\title{
Subcritical Approximation of the Sobolev Quotient and a Related Concentration Result
}

\author{
Giampiero PaLATUCCI
}

ABSTRACT - Let $\Omega$ be a general, possibly non-smooth, bounded domain of $\mathbb{R}^{N}, N \geq 3$. Let $2^{*}=2 N /(N-2)$ be the critical Sobolev exponent. We study the following variational problem

$$
S_{\varepsilon}^{*}=\sup \left\{\int_{\Omega}|u|^{2^{*}-\varepsilon} d x: \int_{\Omega}|\nabla u|^{2} d x \leq 1, u=0 \text { on } \partial \Omega\right\},
$$

investigating its asymptotic behavior as $\varepsilon$ goes to zero, by means of $\Gamma^{+}$-convergence techniques. We also show that sequences of maximizers $u_{\varepsilon}$ concentrate energy at one point $x_{0} \in \bar{\Omega}$.

\section{Introduction.}

Let $\Omega$ be a general bounded, possible non-smooth, bounded open set in $\mathbb{R}^{N}, N \geq 3$. Consider the elliptic Dirichlet problem with nearly critical nonlinearity

$$
\left\{\begin{array}{cl}
-\Delta u_{\varepsilon}=\lambda u_{\varepsilon}^{2^{*}-1-\varepsilon} & \text { in } \Omega, \\
u_{\varepsilon}>0 & \text { in } \Omega, \\
u_{\varepsilon}=0 & \text { on } \partial \Omega,
\end{array}\right.
$$

where $2^{*}=2 N /(N-2), \lambda=N(N-2)$. We know that when $\varepsilon>0$ the above problem has a solution $u_{\varepsilon}$, but when $\varepsilon=0$ it becomes delicate. In particular, the existence and the properties of the solutions strongly depend on the domain $\Omega$ (see $[14,9,2,5]$ and others).

Indirizzo dell'A.: LATP, Université Aix-Marseille III, Faculté des Sciences et Techniques Saint-Jérôme, Avenue Escadrille Normandie-Niémen, 13397 Marseille cedex 20, France

E-mail: giampiero.palatucci@univ-cezanne.fr 
In view of this qualitative change when $\varepsilon=0$, it is interesting to analyze the asymptotic behavior of the subcritical solutions $u_{\varepsilon}$ of (1.1) as $\varepsilon$ goes to zero.

In the case of smooth domains $\Omega$, this analysis was carefully investigated by Han [8] and Rey [15]. They showed that the solutions $u_{\varepsilon}$ of (1.1), that are maximizing for the following variational problem

$$
\begin{gathered}
S_{\varepsilon}^{*}:=\sup \left\{F_{\varepsilon}(u): \int_{\Omega}|\nabla u|^{2} d x \leq 1, u=0 \text { on } \partial \Omega\right\} \\
\text { with } F_{\varepsilon}(u)=\int_{\Omega}|u|^{2^{*}-\varepsilon} d x,
\end{gathered}
$$

concentrate at exactly one point $x_{0}$ in $\bar{\Omega}$; i.e., the measures $\left|\nabla u_{\varepsilon}\right|^{2} d x$ converge (up to subsequences) in the sense of measures to a Dirac mass at $x_{0} \in \bar{\Omega}$. They also showed that $x_{0}$ is a critical point of the Robin function of $\Omega$ (the diagonal of the regular part of the Green function), answering to a conjecture by Brezis and Peletier in [3], in which was analyzed the same problem in the case of $\Omega$ being a spherical domain.

In order to obtain this concentration result, even without localizing the blowing-up, the cited authors also utilize some standard elliptic regularity techniques that require to work in smooth domains.

We also stress that regularity assumptions on the domain $\Omega$ have a strong impact on concentration results. In fact, in the case of smooth domains, the Robin function has no critical points in a neighborhood of the boundary and thus in [3, 8, 15] concentration may occur only in $\Omega$. In [7], Flucher, Garroni and Müller were able to construct an example of a nonsmooth domain $\tilde{\Omega}$, with its Robin function achieving its infimum on the boundary; and then Pistoia and Rey showed that concentration can occur on the boundary, analyzing the asymptotic behavior of the maximizing solutions of the elliptic Dirichlet problem (1.2) in such $\tilde{\Omega}$ (see [13]).

Here we can obtain the same concentration result in general bounded domain, not depending from any strong hypotheses of regularity, as a consequence of Lions' Concentration-compactness principle ([10]) together with the convergence of $\max F_{\varepsilon}$ to $\sup F_{0}$ (with $F_{0}(u)=\int_{\Omega}|u|^{2^{*}} d x$; see
Section 2 ).

We also investigate the asymptotic behavior as $\varepsilon \rightarrow 0$ of the subcritical Sobolev quotient $S_{\varepsilon}^{*}$ in (1.2) by means of De Giorgi's $\Gamma^{+}$-convergence techniques. We can find this kind of approach, that is using $\Gamma^{+}$-convergence techniques to study concentration phenomena linked to critical 
Sobolev exponent, in [1], where Amar and Garroni, following Flucher and Müller in [6], studied the variational problem

$$
\begin{gathered}
S_{\varepsilon}^{g}=\sup \left\{G_{\varepsilon}(u): u \in H_{0}^{1}(\Omega),\|\nabla u\|_{L^{2}(\Omega)} \leq 1\right\} \\
\text { with } G_{\varepsilon}(u)=\varepsilon^{-2^{*}} \int_{\Omega} g(\varepsilon u) d x,
\end{gathered}
$$

where $g$ is a non-negative upper semi-continuous function bounded from above by $c|t|^{2^{*}}$. They obtained a $\Gamma^{+}$-convergence result, that implies concentration phenomena arising in critical growth problems.

Although it is not possible to reduce our problem (1.2) to (1.4), the $\Gamma^{+}$convergence of the functional $F_{\varepsilon}$ is related to the $\Gamma^{+}$-convergence of $F_{0}$ to its upper semi-continuous envelope $\operatorname{sc}^{+} F_{0}$, obtained by Amar and Garroni in [1] (see Section 3).

A fundamental key point of the $\Gamma^{+}$-convergence is specifying the setting for the limit functional, with the choice of the topology. Here, in particular, the topology has to be sufficiently weak to assure the convergence of maximizing sequences and sufficiently strong to allow us to find the concentration.

Due to the presence of the constraint on the Dirichlet energy, the variational problem (1.2) suggests to study every sequence $u_{\varepsilon}$ weakly converging to some function $u$ in the Sobolev space $H_{0}^{1}(\Omega)$. This convergence is not sufficient to describe the problem, so we also have to study every sequence $\left|\nabla u_{\varepsilon}\right|^{2} d x$ converging to some measure $\mu$ in the sense of measures.

We will show that the asymptotic behavior of the functional (1.3), in term of $\Gamma^{+}$-convergence, is described by the following functional $F$ that depends by the two variables $u$, belonging to $H_{0}^{1}(\Omega)$, and $\mu$, being in $\mathcal{M}(\bar{\Omega})$, the set of non-negative bounded total variation Borel measures, with its atomic coefficients $\mu_{i}$ :

$$
F(u, \mu)=\int_{\Omega}|u|^{2^{*}} d x+S^{*} \sum_{i=0}^{\infty} \mu_{i}^{2^{*}}
$$

where $S^{*}$ is the best Sobolev constant of the embedding $H_{0}^{1}(\Omega) \hookrightarrow L^{2^{*}}(\Omega)$, that is

$$
S^{*}=\sup \left\{\int_{\Omega}|u|^{2^{*}} d x: \int_{\Omega}|\nabla u|^{2} d x \leq 1, u=0 \text { on } \partial \Omega\right\} .
$$


Now, a natural question arises: can we localize the blowing up? Our hope is that it will possible to prove that $x_{0}$ is a "harmonic center" of $\Omega$, that is the minimum of the Robin function of $\Omega$. We could work by means of a reasonable second order development of the $\Gamma^{+}$-limit, in order to obtain more informations about the asymptotic behavior of the maximizing sequences. We surely need to look at some techniques introduced in [6] and [7], where Flucher, Garroni and Müller showed that the concentration at the critical points of the Robin function is a very general phenomenon.

Second, again using a similar variational approach, in [11] the author generalizes the asymptotic analysis of subcritical solutions of the analogous problem to (1.1) for the $p$-Laplacian operator.

Finally, in a work in progress with A. Pisante ([12]) we study non-local concentration phenomena in the same spirit of this paper.

The remaining of the paper is divided in two sections. Section 2 is devoted to the concentration result. In Section 3 we state and prove the $\Gamma^{+}$. convergence result.

\section{The concentration result.}

In this section, we will analyze the asymptotic behavior of the sequences $u_{\varepsilon}$ that are maximizers for the nearly critical Sobolev quotient $S_{\varepsilon}^{*}$, as stated in the following theorem.

THEOREM 2.1. Let $u_{\varepsilon}$ be a maximizer sequence for the Sobolev quotient $S_{\varepsilon}^{*}$ defined in (1.2). Then $u_{\varepsilon}$ concentrates at some $x_{0} \in \bar{\Omega}$, i.e.,

$$
\left|\nabla u_{\varepsilon}\right|^{2} d x \stackrel{*}{\rightarrow} \delta_{x_{0}} \text { in } \mathcal{M}(\bar{\Omega}) .
$$

This result is a consequence of Lions's Concentration-compactness principle (see forthcoming Lemma 2.2) together with the convergence of $S_{\varepsilon}^{*}$ to $S^{*}$ as $\varepsilon$ goes to zero (see Proposition 2.4).

LEMma 2.2 ([10, Lemma I.1]). Let $\Omega \subset \mathbb{R}^{N}$ be an open subset and let $u_{n}$ be a sequence in $H_{0}^{1}(\Omega)$ weakly converging to $u$ as $n \rightarrow \infty$ and such that

$$
\left|\nabla u_{n}\right|^{2} d x \stackrel{*}{\rightarrow} \mu \quad \text { and }\left|u_{n}\right|^{2^{*}} d x \stackrel{*}{\rightarrow} v \text { in } \mathcal{M}(\bar{\Omega}) .
$$

Then, either $u_{n} \rightarrow u$ in $L^{2^{*}}(\Omega)$ or there exists a finite set of distinct points $x_{0}, \ldots, x_{k}$ in $\bar{\Omega}$ and positive numbers $v_{0}, \ldots, v_{k}$ such that we have

$$
v=|u|^{2^{*}} d x+\sum_{i=0}^{k} v_{i} \delta_{x_{i}}, \quad v_{i}^{2}\left(S^{*}\right)^{2^{*}} \geq\left(S^{*}\right)^{2} .
$$


Moreover, there exist a positive measure $\tilde{\mu} \in \mathcal{M}(\Omega)$ with spt $\tilde{\mu} \subset \bar{\Omega}$ and positive numbers $\mu_{1}, \ldots, \mu_{k}$ such that

$$
\mu=|\nabla u|^{2} d x+\tilde{\mu}+\sum_{i=0}^{k} \mu_{i} \delta_{x_{i}}, \quad v_{i} \leq S^{*} \mu_{i}^{2^{2}} .
$$

Armed with the concentration-compactness alternative, we can describe the asymptotic behavior of the optimal sequences $u_{n}$ of the critical Sobolev quotient $S^{*}$. The key of the proof is the well-known convexity argument by Lions (see also [6, Lemma 10]).

COROLlary 2.3. Let $u_{n}$ be a maximizing sequence for the critical Sobolev quotient $S^{*}$ defined in (1.7). Then $u_{n}$ concentrates at one point $x_{0} \in \bar{\Omega}$.

Now, we will prove that the subcritical Sobolev quotients $S_{\varepsilon}^{*}$ converge to $S^{*}$ as $\varepsilon$ goes to zero.

Proposition 2.4. For every $\varepsilon>0$, let $S_{\varepsilon}^{*}$ be defined by (1.2) and let $S^{*}$ be defined by (1.7). Then

$$
\lim _{\varepsilon \rightarrow 0} S_{\varepsilon}^{*}=S^{*}
$$

Proof. First, using the fact that $\Omega$ is bounded together with Hölder inequality, we can show that

$$
\limsup _{\varepsilon \rightarrow 0} S_{\varepsilon}^{*} \leq S^{*}
$$

Take $u_{\varepsilon} \in H_{0}^{1}(\Omega)$ maximizers for $S_{\varepsilon}^{*}$; we have

$$
\begin{aligned}
S_{\varepsilon}^{*}=F_{\varepsilon}\left(u_{\varepsilon}\right) & =\int_{\Omega}\left|u_{\varepsilon}\right|^{2^{*}-\varepsilon} d x \\
& \leq\left(\int_{\Omega}\left|u_{\varepsilon}\right|^{2^{*}}\right)^{\frac{2^{*}-\varepsilon}{2^{*}}}|\Omega|^{\frac{\varepsilon}{2^{*}}} \\
& \leq\left(S^{*}\right)^{\frac{2^{*}-\varepsilon}{2^{*}}}|\Omega|^{\frac{\varepsilon}{2^{*}}}
\end{aligned}
$$

Thus, passing to the limit as $\varepsilon$ goes to zero, it follows inequality (2.2).

The remaining inequality easily follows by the pointwise convergence of $F_{\varepsilon}$ to $F_{0}$. This is a standard argument. For every $\delta>0$ there exists 
$u_{\delta} \in H_{0}^{1}(\Omega)$ such that $\left\|\nabla u_{\delta}\right\|_{L^{2}(\Omega)}^{2} \leq 1$ and

$$
F_{0}\left(u_{\delta}\right)>S^{*}-\delta
$$

Clearly, for such function $u_{\delta}$, we have

$$
S_{\varepsilon}^{*} \geq F_{\varepsilon}\left(u_{\delta}\right) .
$$

Then, combining the above inequality with (2.3) and passing to the limit as $\varepsilon$ goes to zero, we get

$$
\begin{aligned}
\liminf _{\varepsilon \rightarrow 0} S_{\varepsilon}^{*} & \geq \lim _{\varepsilon \rightarrow 0} F_{\varepsilon}\left(u_{\delta}\right) \\
& =F_{0}\left(u_{\delta}\right) \\
& \geq S^{*}-\delta
\end{aligned}
$$

that implied the desired inequality thanks to the arbitrariness of $\delta$. The proof is complete.

Completion of THE PROOF OF THEOREM 2.1. The concentration result for the sequence $u_{\varepsilon}$ of maximizers of $S^{*}$ is immediate. Thanks to Proposition 2.4 the maximizers $u_{\varepsilon}$ of $S_{\varepsilon}^{*}$ are a maximizing sequence for $F_{0}$. Hence, Corollary 2.3 ensures that $\left|\nabla u_{\varepsilon}\right|^{2} d x$ converge to a Dirac mass in the sense of measures.

\section{The $\Gamma^{+}$-convergence result.}

The goal of the present section is to describe by means of $\Gamma^{+}$-convergence the asymptotic behavior as $\varepsilon$ goes to zero of the functionals $F_{\varepsilon}$ defined by

$$
F_{\varepsilon}(u)=\int_{\Omega}|u|^{2^{*}-\varepsilon} d x, \quad \forall u \in H_{0}^{1}(\Omega) \text { such that }\|\nabla u\|_{L^{2}(\Omega)}^{2} \leq 1 .
$$

In order to apply $\Gamma^{+}$-convergence, we have to analyze the asymptotic behavior of the sequence $F_{\varepsilon}\left(u_{\varepsilon}\right)$ for every sequence $u_{\varepsilon}$ such that $\left\|\nabla u_{\varepsilon}\right\|_{L^{2}(\Omega)}^{2} \leq 1$. The constraint on the Dirichlet energy of $u_{\varepsilon}$ implies that there exists $\mu \in \mathcal{M}(\bar{\Omega})$ such that $\mu(\bar{\Omega}) \leq 1$ and $\left|\nabla u_{\varepsilon}\right|^{2} d x \stackrel{*}{\rightarrow} \mu$ in $\mathcal{M}(\bar{\Omega})$ and, by Sobolev embedding, there exists $u \in H_{0}^{1}(\Omega)$ such that (up to subsequences) $u_{\varepsilon} \rightarrow u$ in $L^{2^{*}}(\Omega)$.

Thanks to the semi-continuity of $L^{2}$-norm, we have $\mu \geq|\nabla u|^{2} d x$, so we can always decompose $\mu$ in such way:

$$
\mu=|\nabla u|^{2} d x+\tilde{\mu}+\sum_{i=0}^{\infty} \mu_{i} \delta_{x_{i}}
$$


where $\mu_{i} \in[0,1]$ and $x_{i} \in \bar{\Omega}$ is such that $x_{i} \neq x_{j}$ if $i \neq j ; \tilde{\mu}$ can be view as the "non-atomic part" of $\mu-|\nabla u|^{2} d x$.

In analogy with [1], this suggests the setting for the limit functional as the space $X$ defined by

$$
X=X(\bar{\Omega}):=\left\{(u, \mu) \in H_{0}^{1}(\Omega) \times \mathcal{M}(\bar{\Omega}): \mu \geq|\nabla u|^{2} d x, \mu(\bar{\Omega}) \leq 1\right\},
$$

endowed with the topology $\tau$ such that

$$
\left(u_{\varepsilon}, \mu_{\varepsilon}\right) \stackrel{\tau}{\rightarrow}(u, \mu) \stackrel{\operatorname{def}}{\Leftrightarrow}\left\{\begin{array}{l}
u_{\varepsilon} \rightarrow u \text { in } L^{2^{*}}(\Omega) \\
\mu_{\varepsilon}{ }^{*} \mu \text { in } \mathcal{M}(\bar{\Omega})
\end{array}\right.
$$

Note that the topology $\tau$ is compact in $X$, then the $\Gamma^{+}$-convergence of functionals in this space implies the convergence of maxima.

We also note that $X$ is the smallest space where we have to set our problem, as stated in the following proposition by Amar and Garroni.

Proposition 3.1 ([1, Proposition 2.3]). Let $(u, \mu) \in X$, then there exists a sequence $u_{\varepsilon}$ in $H_{0}^{1}(\Omega)$ such that, for every $\varepsilon>0, \int_{\Omega}\left|\nabla u_{\varepsilon}\right|^{2} d x \leq 1$ and
$\left(u_{\varepsilon},\left|\nabla u_{\varepsilon}\right|^{2} d x\right) \stackrel{\tau}{\rightarrow}(u, \mu)$ when $\varepsilon \rightarrow 0$.

Once we have taken the right space, we need to extend our functional $F_{\varepsilon}$ to the whole space $X$ (and we keep the same symbol), in the natural way as follows

$$
F_{\varepsilon}(u, \mu):= \begin{cases}\int_{\Omega}|u|^{2^{*}-\varepsilon} d x & \text { if }(u, \mu) \in X: \mu=|\nabla u|^{2} d x \\ 0 & \text { otherwise in } X .\end{cases}
$$

We prove the existence of the $\Gamma^{+}$-limit of the sequence $F_{\varepsilon}$, giving its characterization in the following theorem.

THEOREM 3.2. There exists the $\Gamma^{+}$-limit $F$ of the sequence of functionals $F_{\varepsilon}$ defined by (3.2) and

$$
F(u, \mu)=\int_{\Omega}|u|^{2^{*}} d x+S^{*} \sum_{i=0}^{\infty} \mu_{i}^{\frac{2^{*}}{2}}, \quad \forall(u, \mu) \in X .
$$

In view of the pointwise convergence of $F_{\varepsilon}$ to $F_{0}$ and the convergence of $S_{\varepsilon}^{*}$ to $S^{*}$ (see Proposition 2.4), Theorem 3.2 can be proved repeating the strategy in the proof of [1, Theorem 3.1]. 
First, we state the $\Gamma^{+}$-convergence result by Amar and Garroni in [1]. Let $g: \mathbb{R} \rightarrow[0, \infty)$ be an upper semi-continuous function such that

(i) $0 \leq g(t) \leq c|t|^{2^{*}}, g \neq 0$ in the $L^{1}$-sense;

(ii) $\exists$ a constant $g_{0}$ such that

$$
\lim _{t \rightarrow 0^{+}} \frac{g(t)}{t^{2^{*}}}=g_{0}
$$

Let $S^{g}$ be the critical generalized Sobolev constant (see [6, Definition 1]) such that

$$
S^{g}=\sup \left\{\int_{\Omega} g(u) d x: u \in H_{0}^{1}(\Omega), \int_{\Omega}|\nabla u|^{2} d x \leq 1\right\} .
$$

For any positive $\varepsilon$, consider the functional $G_{\varepsilon}$ defined in $H_{0}^{1}(\Omega) \times \mathcal{M}(\bar{\Omega})$ by

$$
G_{\varepsilon}(u, \mu)= \begin{cases}\varepsilon^{-2^{*}} \int_{\Omega} g(\varepsilon u) d x & \text { if }(u, \mu) \in X \text { and } \mu=|\nabla u|^{2} d x, \\ 0 & \text { otherwise in } X .\end{cases}
$$

The $\Gamma^{+}$-limit of the functional $G_{\varepsilon}$ with respect to the topology $\tau$ is given in the following theorem.

Theorem 3.3 ([1, Theorem 3.1]). There exists the $\Gamma^{+}$-limit $G$ of the sequence of functionals $G_{\varepsilon}$ and

$$
G(u, \mu)=g_{0} \int_{\Omega}|u|^{2^{*}} d x+S^{g} \sum_{i=0}^{\infty} \mu_{i}^{\frac{2^{*}}{2}},
$$

for every $(u, \mu) \in X$.

We note that in the particular case of $g$ being the critical power $2^{*}$; i.e., $g(t)=|t|^{2^{*}}, \forall t \in \mathbb{R}$, the definitions in (3.3), (3.4), (3.5) and (3.6) become respectively

$$
g_{0}=1, S^{g}=S^{*}, G_{\varepsilon}=F_{0} \text { and } G=F
$$

and then, applying Theorem 3.3, it follows

$$
F_{0} \stackrel{\Gamma^{+}}{\rightarrow} \mathrm{sc}^{+} F_{0} \equiv F,
$$

where $F_{0}$ is extended to the whole space $X$ in the same way as in definition (3.2) and we denote by sc${ }^{+} F_{0}$ the upper semi-continuous envelope of $F_{0}$ with respect to the topology $\tau$. 
Using the convexity argument by Lions together with the fact that the critical Sobolev inequality is strict in bounded domains, Amar and Garroni also provide an optimal upper bound inequality for the limit functional $G$.

Lemma 3.4 ([1, Lemma 3.6]). For every $(u, \mu) \in X$, we have

$$
G(u, \mu) \leq S^{g}
$$

and the equality holds if and only if $(u, \mu)=\left(0, \delta_{x_{0}}\right)$ for some $x_{0} \in \bar{\Omega}$.

Proof of Theorem 3.2. Let us introduce the $\Gamma^{+}$-lim sup and the $\Gamma^{+}$$\liminf$ of $F_{\varepsilon}$, respectively defined for every $(u, \mu) \in X$ by

$$
F^{+}(u, \mu)=\sup \left\{\limsup _{\varepsilon \rightarrow 0} F_{\varepsilon}\left(u_{\varepsilon}, \mu_{\varepsilon}\right):\left(u_{\varepsilon}, \mu_{\varepsilon}\right) \stackrel{\tau}{\rightarrow}(u, \mu)\right\}
$$

and

$$
F^{-}(u, \mu)=\sup \left\{\liminf _{\varepsilon \rightarrow 0} F_{\varepsilon}\left(u_{\varepsilon}, \mu_{\varepsilon}\right):\left(u_{\varepsilon}, \mu_{\varepsilon}\right) \stackrel{\tau}{\rightarrow}(u, \mu)\right\} .
$$

We can write the notion of $\Gamma^{+}$-convergence in terms of the $\Gamma^{+}$-limsup and the $\Gamma^{+}$-liminf (see [4] for further details). The $\Gamma^{+}$-limit $F$ exists if and only if $F^{-}=F^{+}$and, in this case, $F=F^{-}=F^{+}$. Since $F^{-} \leq F^{+}$always holds, in order to prove Theorem 3.2 it is enough to show the following inequalities

$$
\begin{aligned}
& F \geq F^{+} \\
& F \leq F^{-} .
\end{aligned}
$$

The first inequality (3.9) easily follows by the Concentration-compactness principle by Lions.

Proposition 3.5. For every $(u, \mu) \in X$, we have

$$
F(u, \mu) \geq \limsup _{\varepsilon \rightarrow 0} F_{\varepsilon}\left(u_{\varepsilon}, \mu_{\varepsilon}\right)
$$

for every $\left(u_{\varepsilon}, \mu_{\varepsilon}\right) \subset X$ such that $\left(u_{\varepsilon}, \mu_{\varepsilon}\right) \stackrel{\tau}{\rightarrow}(u, \mu)$.

Proof. Let $\left(u_{\varepsilon}, \mu_{\varepsilon}\right)$ be in $X$ such that $\left(u_{\varepsilon}, \mu_{\varepsilon}\right) \stackrel{\tau}{\rightarrow}(u, \mu)$; i.e., $u_{\varepsilon} \rightarrow u$ in $L^{2^{*}}(\Omega)$ and $\left|\nabla u_{\varepsilon}\right|^{2} d x \stackrel{*}{\rightarrow} \mu=|\nabla u|^{2} d x+\tilde{\mu}+\sum_{i=0}^{\infty} \mu_{i} \delta_{x_{i}}$ in $\mathcal{M}(\bar{\Omega})$, 
By Lemma 2.2, it follows that

$$
\left|u_{\varepsilon}\right|^{2^{*}} d x \stackrel{*}{\rightarrow} v=|u|^{2^{*}} d x+\sum_{i=0}^{k} v_{i} \delta_{x_{i}}, \quad \text { with } \quad v_{i} \leq S^{*} \mu_{i}^{\frac{2^{*}}{2}}
$$

Using Hölder Inequality by arguing as in the proof of Proposition 2.4, we have

$$
\begin{aligned}
\limsup _{\varepsilon \rightarrow 0} F_{\varepsilon}\left(u_{\varepsilon}, \mu_{\varepsilon}\right) & \leq \limsup _{\varepsilon \rightarrow 0}\left(\left(\int_{\Omega}\left|u_{\varepsilon}\right|^{2^{*}} d x\right)^{\frac{2^{*}-\varepsilon}{2^{*}}}|\Omega|^{\frac{\varepsilon}{2^{*}}}\right) \\
& \leq v(\bar{\Omega}) \\
& \leq \int_{\Omega}|u|^{2^{*}} d x+S^{*} \sum_{i=0}^{\infty} \mu_{i}^{\frac{2^{*}}{2}} \equiv F(u, \mu),
\end{aligned}
$$

where we used (3.11).

The remaining inequality (3.10) can be obtained like in the proof of [1, Theorem 3.1]. For the sake of self-containment, we will sketch here the proof.

According to the idea of Amar and Garroni, the $\Gamma^{+}$-liminf inequality will be proved in two separate cases: $(u, \mu)=\left(u,|\nabla u|^{2} d x+\tilde{\mu}\right)$ and $(u, \mu)=\left(0, \sum_{i} \mu_{i} \delta_{x_{i}}\right)$. This decomposition is the key point in the proof. In fact, we will be able to use strong $L^{2^{*}}$ convergence on the pairs of the first type (see the forthcoming Proposition 3.6, in which we use $F_{\varepsilon} \rightarrow F_{0}$ ); while on the pairs with purely atomic measure part, the functionals $F_{\varepsilon}$ are local, so we can compute their limit on each single Dirac mass (see Proposition 3.7, in which we use $S_{\varepsilon}^{*} \rightarrow S^{*}$ ). Finally, Theorem 3.2 will be recovered thanks to a unifying lemma (see Lemma 3.8).

Proposition 3.6. Let $(u, \mu) \in X$ be such that $\mu=|\nabla u|^{2} d x+\tilde{\mu}$. Then

$$
F(u, \mu) \leq \liminf _{\varepsilon \rightarrow 0} F_{\varepsilon}\left(u_{\varepsilon}, \mu_{\varepsilon}\right)
$$

for every sequence $\left(u_{\varepsilon}, \mu_{\varepsilon}\right) \subset X$ such that $\left(u_{\varepsilon}, \mu_{\varepsilon}\right) \stackrel{\tau}{\rightarrow}(u, \mu)$ as $\varepsilon \rightarrow 0$.

Proof. We take $\left(u_{\varepsilon}, \mu_{\varepsilon}\right) \subset X$ such that $\mu_{\varepsilon}=\left|\nabla u_{\varepsilon}\right|^{2} d x$ and $\left(u_{\varepsilon}, \mu_{\varepsilon}\right) \stackrel{\tau}{\rightarrow}(u, \mu)$ as $\varepsilon \rightarrow 0$. Since the atomic part of $\mu_{\varepsilon}$ is zero, $u_{\varepsilon} \rightarrow u$ in $L^{2^{*}}(\Omega)$ as $\varepsilon \rightarrow 0$, so (up to a subsequence) $u_{\varepsilon} \rightarrow u$ a.e. in $\Omega$. 
It follows that for almost every $x$ in $\Omega$

$$
\left|u_{\varepsilon}(x)\right|^{2^{*}-\varepsilon} \rightarrow|u(x)|^{2^{*}} \text { as } \varepsilon \rightarrow 0 .
$$

Hence, by Lebesgue Convergence Theorem, we have:

$$
\lim _{\varepsilon \rightarrow 0} \int_{\Omega}\left|u_{\varepsilon}\right|^{2^{*}-\varepsilon} d x=\int_{\Omega}|u|^{2^{*}} d x \equiv F(u, \mu),
$$

that gives the desired inequality. Proposition 3.7. For every $(u, \mu) \in X$ such that $(u, \mu)=\left(0, \sum_{i=0}^{k} \mu_{i} \delta_{x_{i}}\right)$,
with $x_{i} \in \bar{\Omega}$, there exists the $\Gamma^{+}$-limit of $\left(F_{\varepsilon}\right)$ and we have

$$
\left(\Gamma^{+}-\lim _{\varepsilon \rightarrow 0} F_{\varepsilon}\right)(u, \mu)=F(u, \mu) .
$$

Proof. First, we prove the following result. For every open set $A \subset \Omega$, for every $x \in \bar{A}$ and for every $(u, \mu) \in X$ such that $(u, \mu)=\left(0, \delta_{x}\right)$, there exists the $\Gamma^{+}$-limit of the sequence $\left(F_{\varepsilon}\right)$ restricted to $A$ and the following equality holds:

$$
\left(\Gamma^{+}-\lim _{\varepsilon \rightarrow 0} F_{\varepsilon}\right)\left(0, \delta_{x} ; \bar{A}\right)=S^{*}
$$

Let us fix $A \subseteq \Omega$ and let $\varepsilon_{h}$ be such that $\varepsilon_{h} \rightarrow 0$ if $h \rightarrow \infty$. By the compactness property of the $\Gamma^{+}$-convergence, it follows that there exists a subsequence (still denoted by $\varepsilon_{h}$ ) and a functional $F_{A}: X(\bar{A}) \rightarrow[0, \infty)$ such that

$$
\left(\Gamma^{+}-\lim _{h \rightarrow \infty} F_{\varepsilon_{h}}\right)(u, \mu ; \bar{A})=F_{A}(u, \mu)
$$

We also have

$$
S_{\varepsilon_{h}}^{*}=\sup _{X(A)} F_{\varepsilon_{h}} \rightarrow \max _{X(A)} F_{A} \text { as } h \rightarrow \infty
$$

and then, recalling Proposition 2.4,

$$
\max _{X(\bar{A})} F_{A}=S^{*} .
$$

By Proposition 3.6 and Lemma 3.4, with $g(t)=|t|^{2^{*}}$ and $\Omega=A$, it follows

$$
F_{A}(u, \mu)<S^{*}, \forall(u, \mu) \neq\left(0, \delta_{\bar{x}}\right), \forall \bar{x} \in \bar{A} .
$$


Combining the above inequality with (3.12), it follows the existence of $\bar{x} \in \bar{A}$ such that

$$
F_{A}\left(0, \delta_{\bar{x}}\right)=S^{*}
$$

and the above equality can be proved by density for every $(u, \mu)=\left(0, \delta_{x}\right)$ with any $x \in \bar{A}$.

Finally, in order to deal with the case $\mu=\sum_{i=0}^{k} \mu_{i} \delta_{x_{i}}$, it suffices to consider the sequence $\left(u_{\varepsilon}, \mu_{\varepsilon}\right)$ such that

$$
u_{\varepsilon}=\sum_{i=0}^{k} \sqrt{\mu_{i}} u_{\varepsilon}^{i}, \quad \mu_{\varepsilon}=\left|\nabla u_{\varepsilon}\right|^{2} d x
$$

where $\left(u_{\varepsilon}^{i},\left|\nabla u_{\varepsilon}^{i}\right|^{2} d x\right) \stackrel{\tau}{\rightarrow}\left(0, \delta_{x_{i}}\right)$ are the recovering sequences given by the previous step. A detailed proof is very well written in [1, Proposition 3.7].

In order to complete the proof of inequality (3.10), we need the following technical lemma by Amar and Garroni, applied to our functionals $F_{\varepsilon}$.

Lemma 3.8 ([1, Lemma 4.1]).If $F(u, \mu) \leq F^{-}(u, \mu)$ for every $(u, \mu) \in X$ such that

(1) $\mu(\bar{\Omega})<1$;

(2) $\mu=|\nabla u|^{2} d x+\tilde{\mu}+\sum_{i=0}^{k} \mu_{i} \delta_{x_{i}}, x_{i} \in \bar{\Omega}$;

(3) $\operatorname{dist}\left(\overline{\operatorname{supp}(|u|+\tilde{\mu})}, \bigcup_{i=0}^{k}\left\{x_{i}\right\}\right)>0$.

Then

$$
F(u, \mu) \leq F^{-}(u, \mu) \text { for every }(u, \mu) \in X .
$$

COMPLETION OF THE PROOF OF THEOREM 3.2. In view of Lemma 3.8, it remains to prove the $\Gamma^{+}$-liminf inequality for every pair $(u, \mu) \in X$ such that $\mu(\bar{\Omega})<1, \mu=|\nabla u|^{2}+\tilde{\mu}+\sum_{i=0}^{k} \mu_{i} \delta_{x_{i}}$ and $\operatorname{dist}\left(\overline{\operatorname{supp}(|u|+\tilde{\mu})}, \bigcup_{i=0}^{k}\left\{x_{i}\right\}\right)>0$.

As a means to do this, it suffices to consider the recovery sequence

$$
\left(u_{\varepsilon}, \mu_{\varepsilon}\right):=\left(u_{\varepsilon}^{A}+u_{\varepsilon}^{B}, \mu_{\varepsilon}^{A}+\mu_{\varepsilon}^{B}\right),
$$

where $\left(u_{\varepsilon}^{A}, \mu_{\varepsilon}^{A}\right)$ and $\left(u_{\varepsilon}^{B}, \mu_{\varepsilon}^{B}\right)$ are given by Proposition 3.6 and Proposition 3.7, respectively (see [1, pag. 17]). The proof is complete.

REMARK 3.9. We can also deduce the concentration result in Theorem 2.1 , by using the $\Gamma^{+}$-convergence of $F_{\varepsilon}$ to $F$. In fact, by Theorem 3.2 and 
$\Gamma^{+}$-convergence properties, we have that every maximizing sequence $\left(u_{\varepsilon},\left|\nabla u_{\varepsilon}\right|^{2} d x\right)$ of $F_{\varepsilon} t$ must converge to a pair $(u, \mu) \in X$ maximizer for $F$ :

$$
\left(u_{\varepsilon},\left|\nabla u_{\varepsilon}\right|^{2} d x\right) \stackrel{\tau}{\rightarrow}(u, \mu), \quad \text { with } F(u, \mu)=\max _{X(\bar{\Omega})} F .
$$

By Lemma 3.4, with $g(t)=|t|^{2^{*}}$, we have $\max _{X \bar{\Omega})} F=F\left(0, \delta_{x_{0}}\right)$, for some $x_{0} \in \bar{\Omega}$.

It follows

$$
\left(u_{\varepsilon},\left|\nabla u_{\varepsilon}\right|^{2} d x\right) \stackrel{\tau}{\rightarrow}\left(0, \delta_{x_{0}}\right),
$$

that is the desired concentration result.

Acknowledgements. I would like to thank Prof. Adriana Garroni for suggesting the study of concentration problems related to critical Sobolev exponent.

\section{REFERENCES}

[1] M. Amar - A. Garroni, $\Gamma$-convergence of concentration problems, Ann. Scuola Norm. Sup. Pisa Cl. Sci., Vol. 2 (1) (2003), pp. 151-179.

[2] A. BAHRI - J. CORON, On a nonlinear elliptic equation involving the critical Sobolev exponent: the effect of the topology of the domain, Comm. Pure Appl. Math., Vol. 41 (1988), pp. 253-294.

[3] H. BREzIS - L. PELETIER, Asymptotic for Elliptic Equations involving critical growth, Partial differential equations and the calculus of variations, Vol. I, Progr. Nonlinear Differential Equations Appl., Birkhäuser Boston, Boston, MA (1989), pp. 149-192.

[4] G. Dal Maso, An introduction to $\Gamma$-convergence, Birkhäuser, Boston, 1992.

[5] W. Ding, Positive solutions of $\Delta u+u^{(n+2) /(n-2)}=0$ on a contractible domain, J. Partial Differential Equations, Vol. 2 (4) (1989), pp. 83-88.

[6] M. Flucher - S. Müller, Concentration of low extremals, Ann. Inst. H. Poincaré Anal. Non Linéaire, Vol. 10 (3) (1999), pp. 269-298.

[7] M. Flucher - A. Garroni - S. MÜller, Concentration of low energy extremals: Identification of concentration points, Calc. Var., Vol. 14 (2002), pp. 483-516.

[8] Z.-C. Han, Asymptotic approach to singular solutions for nonlinear elliptic equations involving critical Sobolev exponent, Ann. Inst. Henri Poincaré, Vol. 8 (2) (1991), pp. 159-174.

[9] J. KAZDAN - F. WARNER, Remarks on some quasilinear elliptic equations, Comm. Pure Appl. Math., Vol. 38 (1975), pp. 557-569.

[10] P. L. Lions, The concentration-compactness principle in the calculus of variations. The limit case, Rev. Mat. Iberoamericana, Vol. 1 (1985), pp. $145-201$. 
[11] G. Palatucci, p-Laplacian problems with critical Sobolev exponent, Asymptotic Analysis, to appear.

[12] G. Palatucci - A. Pisante, Sobolev embeddings and concentration-compactness alternative for fractional Sobolev spaces, submitted paper, available online at http://mipa.unimes.fr/preprints.html.

[13] A. Pistoia - O. ReY, Boundary blow-up for a Brezis-Peletier problem on a singular domain, Calc. Var. Partial Differential Equations, Vol. 18 (3) (2003), 243-251.

[14] S. Pohozaev, Eigenfunctions of the Equations $\Delta u=\lambda f(u)$, Soviet Math. Dkl., Vol. 6 (1965), pp. 1408-1411.

[15] O. Rey, Proof of the conjecture of H. Brezis and L. A. Peletier, Manuscripta math., Vol. 65 (1989), pp. 19-37.

Manoscritto pervenuto in redazione il 7 dicembre 2009. 